\title{
Dynamics of neutral molecules stored in a ring
}

\author{
Floris M. H. Crompvoets, Hendrick L. Bethlem, Jochen Küpper, André J. A. van Roij, and Gerard Meijer \\ Fritz-Haber-Institut der Max-Planck-Gesellschaft, Faradayweg 4-6, D-14195 Berlin, Germany; \\ FOM-Institute for Plasmaphysics Rijnhuizen, Edisonbaan 14, NL-3439 MN Nieuwegein, The Netherlands; \\ and Department of Molecular and Laser Physics, University of Nijmegen, Toernooiveld 1, \\ NL-6525 ED Nijmegen, The Netherlands
}

(Received 24 December 2003; published 7 June 2004)

\begin{abstract}
A decelerated beam of neutral ammonia molecules is injected into an electrostatic storage ring. Electrostatic lenses are used to map the emittance of the decelerator onto the acceptance of the ring. The tangential velocity spread of the package of molecules in the ring is set to less than $1 \mathrm{~m} / \mathrm{s}$. The package of molecules can be observed for more than 50 distinct round trips, corresponding to $40 \mathrm{~m}$ in circular orbit and almost $0.5 \mathrm{~s}$ storage time, sufficiently long for a first investigation of its transversal motion in the ring.
\end{abstract}

DOI: 10.1103/PhysRevA.69.063406

PACS number(s): 33.80.Ps, 29.20.Dh, 33.55.Be, 39.10.+j

Getting full control over both the internal and external degrees of freedom of molecules has been an important goal in molecular physics during the past decade. This control can be achieved, in principle, with appropriately tailored electromagnetic fields [1,2]. Radiation fields can be used, for instance, to prepare molecules in a single selected quantum state, thereby controlling their electronic, vibrational, and rotational degrees of freedom. Static inhomogeneous electric and magnetic fields have been used for a long time to (de)focus polar molecules in a beam, thereby manipulating their transversal position and velocity and controlling their orientation [3]. Time-varying electric fields can be used to efficiently change the forward (longitudinal) position and velocity of these molecules as well [4,5]. In the experiments reported here, a package of ammonia molecules in a single rovibrational state, spatially oriented, is decelerated and focused and subsequently forced in circular orbits. The absolute velocity, as well as the width of the velocity distribution, of the package of molecules is under computer control, and these experiments thereby serve as a demonstration of the level of control over neutral molecules that can nowadays be achieved.

Full control over the internal and external degrees of freedom of molecules is essential in the presently very active field of cold molecules. Trapped samples of neutral molecules have been created by means of buffer gas cooling in a magnetic trap [6], by using Stark deceleration of a molecular beam in combination with an electrostatic trap [7], and by pairing cold atoms to form molecules in an optical [8] or magnetic trap [9]. Recently, spectacular progress has been made with association of ultracold atoms assisted by magnetically induced Feshbach resonances, resulting in the first molecular Bose-Einstein condensates [10-13]. In the field of cold molecules there is a particular interest in cold dipolar molecules which stems from the presence of the anisotropic, long-range dipole-dipole interaction in these samples, which is predicted to lead to interesting physics and novel applications [14].

A beam of polar molecules can be state-selected and focused with an electrostatic hexapole lens. By bending a hexapole into a torus, a storage ring for neutral molecules can be created [15]. Recently, we have experimentally dem- onstrated trapping of packages of decelerated ammonia molecules in such a prototype electrostatic storage ring [16]. A potential advantage of a storage ring over a trap is that the molecules can interact repeatedly, at distinct positions and at well-defined times, with electromagnetic fields and/or other particles. This can be used, for instance, to study ultracold collisions and reactions $[17,18]$. In such experiments, the effect of weak interactions can be enhanced by increasing the storage time in the ring, i.e., by accumulating weak effects during many round trips. It is also conceivable to dedicate a section of the ring to evaporative cooling of molecules. Yet another way to cool molecules and to create a high brightness molecular beam might be stochastic cooling, which has been applied successfully in storage rings for charged particles [19].

Inhomogeneous time-varying electric fields can be used to manipulate the distribution of polar molecules in phase space; appropriately designed electric fields rotate the distribution in phase space uniformly, thereby either minimizing the spatial spread at a certain position (longitudinal focusing or bunching) or minimizing the longitudinal velocity spread (longitudinal cooling or bunch rotation). The operation principle of such a buncher for neutral molecules has been detailed elsewhere [5]. To be able to inject packages of molecules with an as low as possible initial temperature into the storage ring, a buncher is installed between the Starkdecelerator and the ring. To further increase the number of captured molecules the transversal emittance of the decelerator is mapped onto the acceptance of the ring with electrostatic hexapole lenses. Together, these elements enable the control over the full 6-dimensional phase space distribution of the package of molecules entering the ring.

The setup, consisting of the injection beamline and the storage ring, is depicted schematically in Fig. 1. The beamline has been described in detail previously [5,20]. Briefly, a beam of deuterated ammonia is formed by expanding a mixture of less than $1 \%{ }^{14} \mathrm{ND}_{3}$ seeded in Xenon through a cooled pulsed valve $\left(-70^{\circ} \mathrm{C}\right)$ into vacuum. The molecular beam has a velocity of approximately $285 \mathrm{~m} / \mathrm{s}$ and a longitudinal temperature of about $1 \mathrm{~K}$. Due to the adiabatic expansion roughly $60 \%$ of all the $\mathrm{ND}_{3}$ molecules in the beam 


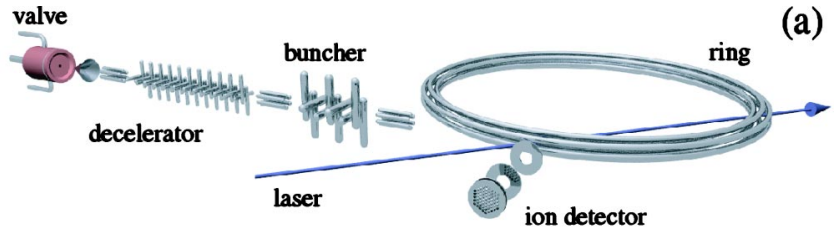

(b)
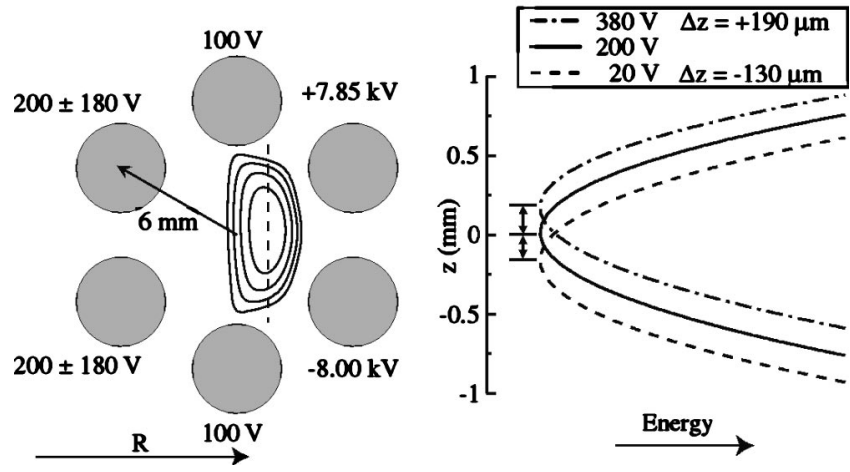

FIG. 1. (Color online) (a) Scheme of the experimental setup. The injection beamline consists of a pulsed valve, a Stark decelerator, hexapoles, and a buncher. (b) The molecules are trapped in a two-dimensional potential well. The center of the ring is to the left. Equipotential lines are shown at $0.02 \mathrm{~cm}^{-1}$ intervals. Molecules are detected in the ring using a uv-laser based ionization detection scheme. The inset shows the vertical position of the potential well along the equilibrium radius for three values of the (ac modulation) voltage on the inner ring electrodes.

reside in the $|J, K\rangle=|1,1\rangle$ inversion doublet, the ground-state level for para-ammonia molecules.

After the beam has passed through a skimmer into a second vacuum chamber the ammonia molecules in low-field seeking states of the upper level of the inversion doublet are transversally focused with a pulsed electrostatic hexapole lens into the Stark decelerator. Molecules in high field seeking states are defocused and consequently lost. The Stark decelerator consists of an array of 64 equidistant electric field stages. Each stage creates a high electric field of $90 \mathrm{kV} / \mathrm{cm}$ on the molecular beam axis. When the ammonia molecules enter theses stages, they will gain Stark energy, which is compensated by a loss in kinetic energy. If the electric field is greatly reduced before the molecules have left this region, they will not regain the lost kinetic energy. The molecules will repeatedly undergo this process while passing through the array of electric field stages, which are switched synchronously with the decelerating package. The molecules are decelerated to $91.8 \mathrm{~m} / \mathrm{s}$ for the experiments described here.

At the exit of the decelerator the calculated longitudinal phase space distribution is $\Delta x \times \Delta v_{x}=[1.1 \mathrm{~mm}] \times[5.9 \mathrm{~m} / \mathrm{s}]$ and the transversal phase space distributions are $\Delta y \times \Delta v_{y}$ $=\Delta z \times \Delta v_{z}=[1.0 \mathrm{~mm}] \times[5.0 \mathrm{~m} / \mathrm{s}]$ where $x$ lies along the molecular beam axis. Here the position spread and velocity spread are the full width at half maximum (FWHM) of a fitted Gaussian distribution. The transversal phase space distribution is mapped onto the acceptance of the ring using a telescope of two electrostatic hexapole lenses. The acceptance of the ring is in fact set by the vertical $(z)$ and radial $(r)$ eigenfrequencies of the potential well which are numerically found as $f_{z}=390 \mathrm{~Hz}$ and $f_{r}=910 \mathrm{~Hz}$ near the minimum of the well. The relation between the frequency $f_{s}$, the accepted position spread $\Delta s$, and the accepted velocity spread $\Delta v_{s}$ is given by $\Delta v_{s}=2 \pi f_{s} \Delta s$. The potential well shown in Fig. 1 contains both the Stark energy and the quasipotential centrifugal energy for molecules moving in circular orbits with a tangential velocity of $91.8 \mathrm{~m} / \mathrm{s}$. It is evident from the equipotential lines in Fig. 1 that the potential well is asymmetric in the dipolar configuration that we have used. To load the ring the hexapoles rotate the transversal phase space distribution of the molecules uniformly such that it matches the vertical acceptance of the ring, because in this direction the potential well is the shallowest. For this, the hexapoles are switched on for only a few tens of microseconds, corresponding to an effective length of a few millimeters for molecules moving with a velocity of $91.8 \mathrm{~m} / \mathrm{s}$. The buncher creates an harmonic potential well that rotates the longitudinal phase space distribution until the longitudinal velocity spread has been minimized. Due to the limited opening angle of the entrance slit of the ring, a part of the package of molecules is cut off by the outer pair of electrodes. The numerically calculated phase space distribution of the package of molecules entering the ring is given by $\Delta r \times \Delta v_{r}=[2.3 \mathrm{~mm}]$ $\times[2.5 \mathrm{~m} / \mathrm{s}], \quad \Delta z \times \Delta v_{z}=[1.4 \mathrm{~mm}] \times[1.4 \mathrm{~m} / \mathrm{s}], \quad \Delta x \times \Delta v_{x}$ $=[9.5 \mathrm{~mm} \times 0.64 \mathrm{~m} / \mathrm{s}]$.

After the molecules have entered the $25-\mathrm{cm}$ diameter electrostatic storage ring, the two outer ring electrodes are rapidly $(<100 \mathrm{~ns})$ switched to high voltages. This creates an electric field that delivers the centripetal force on the molecules. Just prior to detection the high voltages on these electrodes are switched off again. Then the ammonia molecules are ionized in a $2+1$ resonance enhanced multiphoton ionization (REMPI) process, using $317 \mathrm{~nm}$ radiation. The uv light is focused and only molecules in the approximately $3 \mathrm{~mm}$ long, $100 \mu \mathrm{m}$ diameter beam waist are detected. The ions are repelled from the storage ring by the residual electric field created by the permanently present $200 \mathrm{~V}$ and $100 \mathrm{~V}$ on the inner and middle ring electrodes, respectively, and are then detected with a linear time-of-flight mass spectrometer.

Figure 2 shows the recorded $\mathrm{ND}_{3}$ parent ion signal as a function of the storage time in the ring; the origin of the time axis is at the time when the high voltages on the ring are switched on. Each data point represents the parent ion signal intensity averaged over 20 laser shots, i.e., averaged over 20 subsequent deceleration, loading and detection cycles. The experiment normally runs at a $10 \mathrm{~Hz}$ repetition rate. For the measurements that exceed $100 \mathrm{~ms}$ storage times in the ring, however, the repetition rate is reduced; the laser system still runs at $10 \mathrm{~Hz}$, but the effective repetition rate is reduced with a mechanical shutter. The ion signal is proportional to the density of ammonia molecules in the ring. The round trip time is $t_{r}=8.6 \mathrm{~ms}$, determined from fitting the peaks of the signal due to the first 18 round trips, and it is seen that the peak intensity after each round trip gradually decreases while the width of the peaks increases. This is a result of the residual tangential velocity spread of the package of molecules in the ring. Molecules with a tangential velocity of $91.8 \mathrm{~m} / \mathrm{s}$ traverse the ring at the equilibrium orbit, which is a distance $r=1.3 \mathrm{~mm}$ away from the center of the hexapole geometry. 


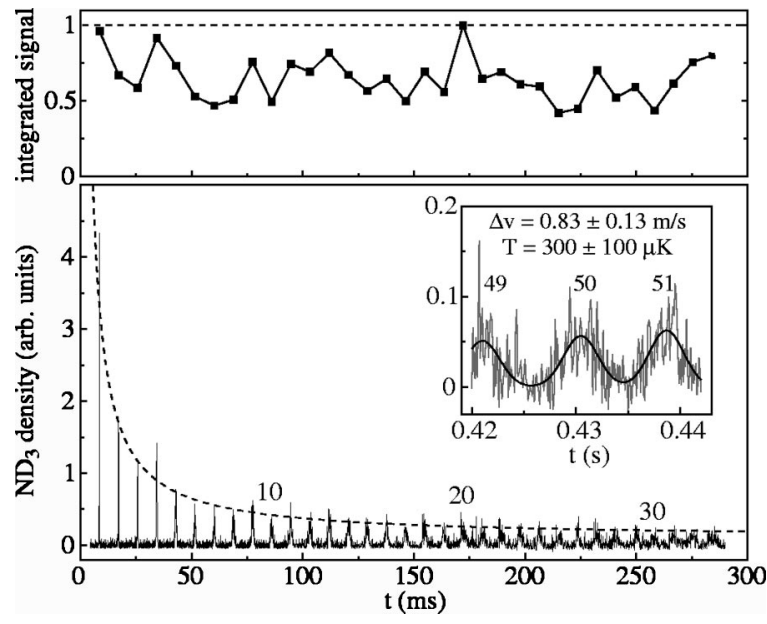

FIG. 2. The solid line in the lower graph shows the ammonia density at the loading position in the ring as a function of storage time. The dashed line is a $1 / t$ plot, used to guide the eye. In the inset, a measurement of the ammonia density after 49-51 round trips is shown, together with a multipeak Gaussian fit, from which a longitudinal temperature of $300 \pm 100 \mu \mathrm{K}$ is deduced for the package of molecules in the ring. The upper graph shows the timeintegrated signal after each round trip.

Faster molecules orbit the ring at a larger radius and hence have a longer flight path; slower molecules orbit at a smaller radius and hence have a shorter flight path. This difference in flight paths between faster and slower molecules counteracts the longitudinal spreading out of the molecular package. As the relative change in orbit radius is small, however, this effect is very limited in the present case and by far not sufficient to make the storage ring isochronous. Therefore the tangential spreading out of the package in the ring can be very well approximated with the longitudinal spreading out of a package in linear free flight. The time-spread of the signal at the $n$th round trip is thus given by $(1 / v) \sqrt{\left(\Delta x_{0}\right)^{2}+\left(\Delta v_{0} n t_{r}\right)^{2}}$, where $\Delta x_{0}$ is the initial longitudinal position spread, $v$ the average velocity, and $\Delta v_{0}$ the longitudinal velocity spread. Without further losses from the ring, the peak density is inversely proportional to this width. When the initial position spread can be neglected, this implies an expected $1 / n$ behavior for the peak intensities, as sketched with the dashed line in Fig. 2. It is seen that the peak heights actually do not decrease monotonically. This is explained by the two-dimensional oscillatory motion of the package of molecules in the ring in combination with the position sensitive detection scheme. The oscillatory motion can be particularly prominent when there is a slight transversal phase space mismatch when the molecules are injected into the ring. In the upper graph of Fig. 2 the time-integrated signal for each round trip is shown. This signal clearly shows the relatively large intensity fluctuations just discussed, but it is also seen that, on average, the signal remains practically constant. Loss due to collisions with background gas can be neglected as the background pressure in the ring chamber is $10^{-9} \mathrm{mbar}$, corresponding to an anticipated $1 / e$ decay time of several seconds. Furthermore, in the dipolar electric field geometry used in this experiment, the molecules are never in zero electric field so zero-field crossing (Majorana) transitions are avoided.

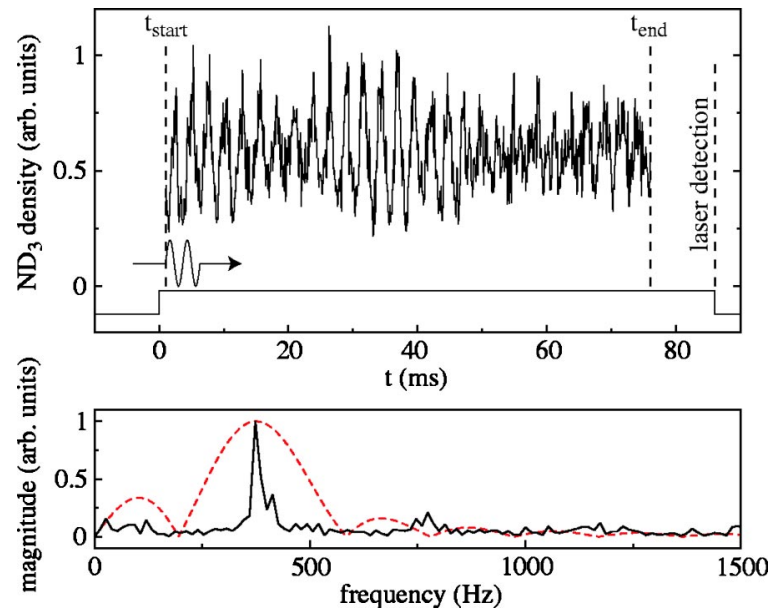

FIG. 3. (Color online) The upper graph shows the density of ammonia molecules in the ring after 10 round trips as a function of the start time of the ac voltage modulation. The modulation signal is a two-period sine wave with a period of $t=2.56 \mathrm{~ms}$. The starting point of this modulation signal is scanned stepwise from $t_{\text {start }}$ to $t_{\text {end }}$. A Fourier transformation of the data is shown in the lower graph (solid curve) together with the frequency spectrum of the two-period sine wave (dashed curve).

The inset of Fig. 2 shows the signal after 49, 50, and 51 round trips. The molecules have then been stored in the ring for about $0.43 \mathrm{~s}$, corresponding to a total flight path of more than $40 \mathrm{~m}$. It is seen from the data that the package of molecules has stretched out so far that it fills about half the ring, i.e., its FWHM length has become about $40 \mathrm{~cm}$. A longitudinal temperature can be deduced from the relative temporal width of the signal from the 50th round trip. After such a long flight distance, the contribution of the initial position spread to the width can be neglected, and the relative temporal width is in a good approximation equal to $\Delta v / v$. From the multipeak Gaussian fit of the data (dark solid line), in combination with the known $91.8 \mathrm{~m} / \mathrm{s}$ tangential velocity of the molecules, the absolute longitudinal velocity spread is found as $0.83 \pm 0.13 \mathrm{~m} / \mathrm{s}$. This corresponds to a longitudinal temperature of $300 \pm 100 \mu \mathrm{K}$, in close agreement with the numerically calculated value at the entrance of the ring.

The transversal motion of the molecules in the ring can be excited by applying an ac voltage on top of the $200 \mathrm{~V}$ dc voltage on the inner ring electrodes. This leads to an oscillatory motion of the potential well, which, for the electric field configuration used, is almost exclusively in the vertical direction, as shown in Fig. 1. In the experiment, the vertical motion of the package of molecules is driven by a twoperiod sine wave with an amplitude of $180 \mathrm{~V}$ and with a period of $t=2.56 \mathrm{~ms}$. This modulation is started at a variable time $t_{\text {start }}$ after the molecules have entered the ring, which is at $t=0$. The block pulse indicates the time that the molecules are confined in the ring. In the upper graph of Fig. 3 the density of ammonia molecules in the ring after ten round trips, i.e., after the molecules have been in the ring for $86 \mathrm{~ms}$, is shown as a function of the time when the ac modulation starts (relative to the switching on of the ring). Each data point represents the averaged signal over 30 laser shots. The ammonia density in the laser detection area after these 
10 round trips shows a strong modulation, the equivalent of a vertical betatron oscillation in charged particle storage rings. In our experimental setup the tightly focused detection laser crosses the ring in the horizontal plane, and vertical oscillations are therefore more readily detected than radial oscillations. Although the ac modulation voltage shakes the potential well only roughly $160 \mu \mathrm{m}$ up and down from the vertical equilibrium position, the driven vertical oscillation of the molecules is calculated to lead to an amplitude of about $1 \mathrm{~mm}$. It is seen from the measurements that even when the ac modulation is induced during the first round trip, the package of molecules coherently oscillates in the vertical direction after 10 round trips. From these measurements, the eigenfrequency for the vertical oscillation in the ring can be accurately determined.

In the lower graph of Fig. 3 a Fourier transformation of the data is shown. The main peak in the frequency spectrum is found at $373 \mathrm{~Hz}$, with a shoulder at $413 \mathrm{~Hz}$, and the first overtone of this peak is visible as well. Also shown in this figure is the frequency spectrum of the two-period sine wave (dashed), which is set to drive the fundamental vertical oscillation. The experimentally determined value of $373 \mathrm{~Hz}$ for this oscillation frequency is close to the calculated value of $f_{z}=390 \mathrm{~Hz}$. When detection would be performed exactly at the center of the vertical motion, the package of molecules would pass the laser focus twice per oscillation and only the overtone would be observed. If the laser detection region would be at an extremum of the vertical motion, only the fundamental frequency would appear. The actual relative intensities of these two frequency components in the spectrum therefore depends on the details of the alignment of the laser relative to the ring as well as of the coupling of the injection beam line to the ring. The envelope of the modulation of the ammonia density appears like a typical beating pattern, indicative of coupled motion at closely spaced oscillation frequencies. In the Fourier transformation this shows up as the $413 \mathrm{~Hz}$ shoulder of the main $373 \mathrm{~Hz}$ resonance. It turns out that, rather than being due to typical beating, the intensity of the envelope of the modulation correlates with the intensity of the corresponding time-integrated signal shown in the upper graph of Fig. 2; the vertical oscillation of the package of molecules shows the largest modulation depth in the detection region when this oscillation is initiated at a time when the package has the best overlap with the detection region.

By optimizing the transversal phase-space matching of the injection beam line onto the storage ring using hexapole lenses and by reducing the longitudinal temperature of the package of molecules prior to injection using a buncher, we have been able to detect ammonia molecules in an $80 \mathrm{~cm}$ circumference storage ring after more than 50 round trips. The ammonia molecules are actually stored in the ring considerably longer than this, but they start to fill up the whole ring with a nearly constant density and the fast molecules from the $n$th round trip can then experimentally no longer be distinguished from the slow molecules in the $(n+1)$ th round trip. The transversal oscillations in the ring can be driven with relatively low ac voltages, and, in combination with position sensitive detection schemes, this can be used for an accurate measurement of the fundamental oscillation frequencies. This opens the way for more detailed studies of the dynamics of polar molecules in an electrostatic storage ring. It is well known that in ion traps, for instance, strong resonances occur when the axial and radial frequencies are in tune [21]. Similar effects will be present in the storage ring for neutral molecules as well, and at certain parametersettings molecular trajectories might become highly unstable. Investigation and a detailed understanding of these ring dynamics is important for the design and construction of future sectional storage rings [22].

This work is part of the research program of the "Stichting voor Fundamenteel Onderzoek der Materie (FOM)," which is financially supported by the "Nederlandse Organisatie voor Wetenschappelijk Onderzoek (NWO)."
[1] P. B. Corkum, C. Ellert, M. Mehendale, P. Dietrich, S. Hankin, S. Aseyev, D. Rayner, and D. Villeneuve, Faraday Discuss. 113, 47 (1999).

[2] H. Stapelfeldt and T. Seideman, Rev. Mod. Phys. 75, 543 (2003).

[3] Atomic and Molecular Beam Methods, edited by G. Scoles (Oxford Universiy Press, New York, 1988, 1992), Vols. 1 and 2.

[4] H. L. Bethlem, G. Berden, and G. Meijer, Phys. Rev. Lett. 83, 1558 (1999).

[5] F. M. H. Crompvoets, R. T. Jongma, H. L. Bethlem, A. J. A. van Roij, and G. Meijer, Phys. Rev. Lett. 89, 093004 (2002).

[6] J. D. Weinstein, R. deCarvalho, T. Guillet, B. Friedrich, and J. M. Doyle, Nature (London) 395, 148 (1998).

[7] H. L. Bethlem, G. Berden, F. M. H. Crompvoets, R. T. Jongma, A. J. A. van Roij, and G. Meijer, Nature (London) 406, 491 (2000).

[8] T. Takekoshi, B. M. Patterson, and R. J. Knize, Phys. Rev.
Lett. 81, 5105 (1998).

[9] N. Vanhaecke, W. de Souza Melo, B. L. Tolra, D. Comparat, and P. Pillet, Phys. Rev. Lett. 89, 063001 (2002).

[10] S. Jochim, M. Bartenstein, A. Altmeyer, G. Hendl, S. Riedl, C. Chin, J. H. Denschlag, and R. Grimm, Science 302, 2101 (2003).

[11] S. Dürr, T. Volz, A. Marte, and G. Rempe, Phys. Rev. Lett. 92, 020406 (2004).

[12] K. Xu, T. Mukaiyama, J. R. Abo-Shaeer, J. K. Chin, D. E. Miller, and W. Ketterle, Phys. Rev. Lett. 91, 210402 (2003).

[13] M. Greiner, C. A. Regal, and D. S. Jin, Nature (London) 426, 537 (2003).

[14] D. DeMille, Phys. Rev. Lett. 88, 067901 (2002).

[15] D. P. Katz, J. Chem. Phys. 107, 8491 (1997).

[16] F. M. H. Crompvoets, H. L. Bethlem, R. T. Jongma, and G. Meijer, Nature (London) 411, 174 (2001).

[17] K. Burnett, P. S. Julienne, P. D. Lett, E. Teisinga, and C. J. Williams, Nature (London) 416, 225 (2002). 
[18] N. Balakrishnan and A. Dalgarno, Chem. Phys. Lett. 341, 652 (2001).

[19] S. van der Meer, Rev. Mod. Phys. 57, 689 (1985).

[20] H. L. Bethlem, F. M. H. Crompvoets, R. T. Jongma, S. Y. T. van de Meerakker, and G. Meijer, Phys. Rev. A 65, 053416
(2002).

[21] R. Alheit, T. Gudjons, S. Kleineidam, and G. Werth, Rapid Commun. Mass Spectrom. 10, 583 (1996).

[22] H. Nishimura, G. Lambertson, J. G. Kalnins, and H. Gould, Rev. Sci. Instrum. 74, 3271 (2003). 